\title{
Axial Relocation Model Extension in Bison
}

K. A. Gamble

August 2018

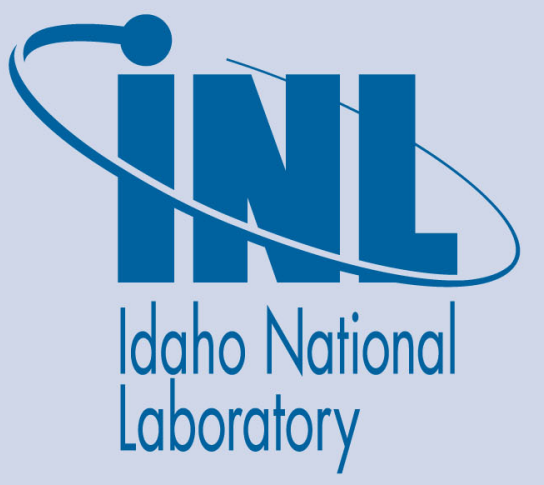

The INL is a U.S. Department of Energy National Laboratory operated by Battelle Energy Alliance 


\title{
Axial Relocation Model Extension in Bison
}

\author{
K. A. Gamble
}

August 2018

Idaho National Laboratory Idaho Falls, Idaho 83415

http://www.inl.gov

Prepared for the

U.S. Department of Energy

Under DOE Idaho Operations Office

Contract DE-AC07-05ID14517 


\section{Axial Relocation Model Extension in Bison L3:FMC.FUEL.17.04}

K. A. Gamble, INL

August 31, 2018
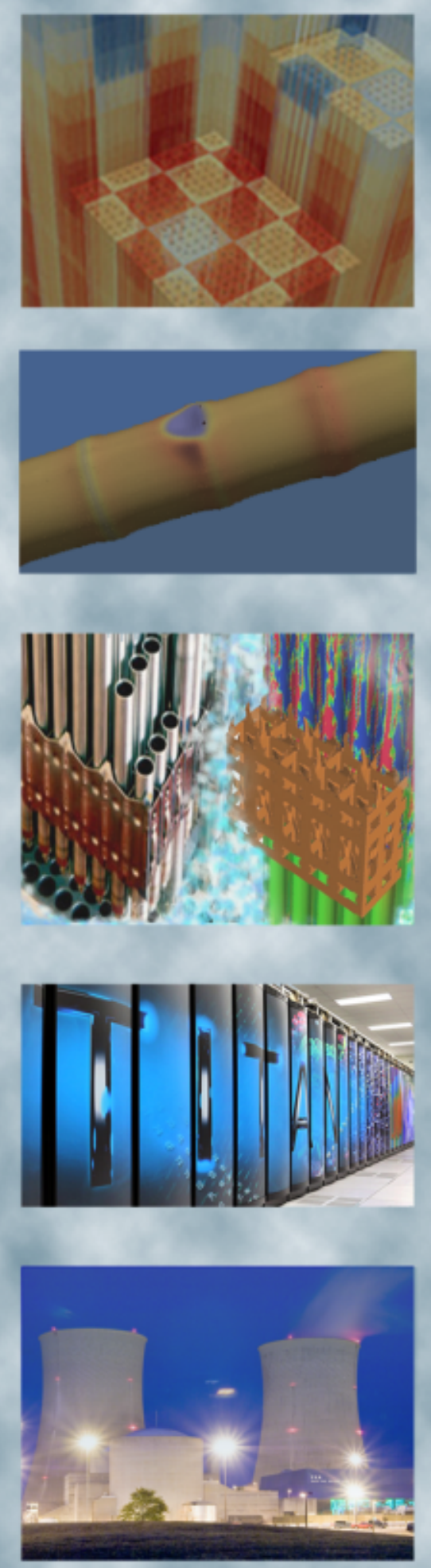
REVISION LOG

\begin{tabular}{|c|c|c|l|}
\hline Revision & Date & Affected Pages & \multicolumn{1}{c|}{ Revision Description } \\
\hline 0 & $8 / 30 / 2018$ & All & Initial Release \\
\hline 1 & $10 / 24 / 2018$ & 13 & Correction in equation \\
\hline & & & \\
\hline & & & \\
\hline
\end{tabular}

\section{Document pages that are:}

Export Controlled NONE

IP/Proprietary/NDA Controlled NONE

Sensitive Controlled NONE

This report was prepared as an account of work sponsored by an agency of the United States Government. Neither the United States Government nor any agency thereof, nor any of their employees, makes any warranty, express or implied, or assumes any legal liability or responsibility for the accuracy, completeness, or usefulness of any information, apparatus, product, or process disclosed, or represents that its use would not infringe privately owned rights. Reference herein to any specific commercial product, process, or service by trade name, trademark, manufacturer, or otherwise, does not necessarily constitute or imply its endorsement, recommendation, or favoring by the United States Government or any agency thereof. The views and opinions of authors expressed herein do not necessarily state or reflect those of the United States Government or any agency thereof.

\section{Requested Distribution:}

To: FMC FA Lead

Copy: CASL PM 


\title{
Axial Relocation Model Extension in Bison
}

\author{
K. A. Gamble \\ Fuels Modeling and Simulation \\ Idaho National Laboratory \\ P.O. Box 1625 \\ Idaho Falls, ID 83415-3840
}

August 31, 2018 


\section{Contents}

\begin{tabular}{ll}
\hline Introduction & 5
\end{tabular}

\begin{tabular}{|lll}
\hline 2 & The Axial Relocation Model & 6
\end{tabular}

3 The Extension of the Axial Relocation Model 11

3.1 Layered Two-Dimensional Framework . . . . . . . . . . . . . . . . . . . . . . . . . . 11

3.2 Gap Calculation in Relocatable Mass Loop . . . . . . . . . . . . . . . . . . . . . . . . . . . . . . 11

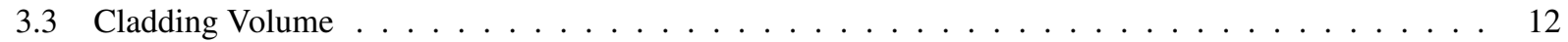

3.4 Mesh Movement in Crumbled Layers $\ldots \ldots \ldots \ldots$

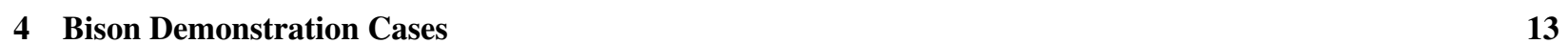

\begin{tabular}{llr}
\hline 5 & Summary & 16
\end{tabular}

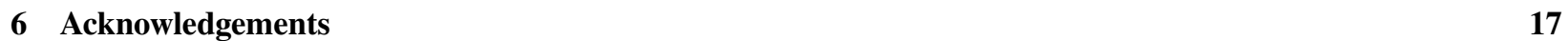




\section{Introduction}

Fuel rods subjected to a Loss of Coolant Accident (LOCA) in a nuclear reactor can undergo a complex process known as fuel fragmentation, relocation, and dispersal (FFRD) [1]. The loss of coolant results in a severe degradation of the thermal heat transfer coefficient on the waterside surface of the cladding. This, coupled with the loss of coolant pressure causes the cladding to distend (balloon) outward. If the cladding distention is large enough, fuel fragmented during irradiation, may relocate from upper regions of the rod into the ballooned region. This mass relocation results in increased localized heating due to the presence of more fuel within the balloon resulting in further clad distention leading to potential rupture. In the event that cladding rupture occurs the relocated fragmented fuel may disperse outside of the rupture opening if the size of the fuel fragments is small enough compared to the rupture opening.

Fuel performance modeling can be used to help understand the behavior of fuel rods during the LOCA transient. Existing models are particularly limited in regards to FFRD. This report briefly describes the existing state of the art for fuel performance modeling of fragmentation and fuel axial relocation. A more in depth description of the axial relocation model implemented into the Bison fuel performance code [2-4] in FY17 is provided. In this report, the extension of this existing model from a layered 1D framework to a 2D framework that allows for the introduction of azimuthal variation in the boundary conditions as experienced during a LOCA is discussed. The 2D framework utilized has been developed by the author of this report as part of his ongoing dissertation research at the University of South Carolina under the direction of Professor Travis W. Knight. The report briefly describes the 2D framework used and highlights in more detail the required developments in Bison to facilitate the extension of the existing axial relocation model to work within this framework. Finally, a few demonstration cases are provided that illustrate the benefit of using the new extended axial relocation model for the simulation of fuel rods under LOCA conditions as well as confirming for symmetric boundary conditions that the extended model calculates the same results as the original. 


\section{The Axial Relocation Model}

In the literature two axial relocation models for fuel performance have been identified. The first, developed by Siefken [5] was based upon the analysis of two experimental LOCA test series completed at the Power Burst Facility in the United States and the FR2 reactor in Germany with a limited burnup range of up to $35 \mathrm{MWd} / \mathrm{kgU}$. In this model the regions of the rod where fuel has been determined to relocate assumes a constant void fraction of 0.3 meaning that regardless of the size of the fuel fragments each layer where mass has relocated to contains $70 \%$ fuel particles and $30 \%$ gas. In reality, the sizes of the fragments can vary wildly resulting in scenarios where the packing fraction of fuel is different than 70\%. Therefore, in Bison the axial relocation model originally implemented based upon the layered 1D formulation was that of Jernkvist and Massih [6].

The model proposed is more sophisticated than Siefken's because it allows for the packing fraction $(\phi)$ to evolve depending upon the irradiation of the fuel. The packing fraction is calculated assuming a binary system of particle sizes that is used to differentiate fuel fragments formed during normal irradiation (fragments) and those that form in high burnup fuel through a process known as pulverization (pulvers).

In the Bison implementation the correlation used for determining the size of fragments is the model proposed by Coindreau et al. [7] which calculates the number of fragments in fresh fuel before including irradiation (burnup) effects through:

$n_{f}^{o}=\max \left(1, \min \left(\frac{7 q_{M}^{\prime}-8}{17}, 16\right)\right)$

$n_{f}=\min \left(n_{f}^{o}+\frac{\left(16-n_{f}^{o}\right) B u_{a v}}{50}, 16\right)$

where $n_{f}^{o}$ is the number of radial fragments expected in fresh fuel subjected to the maximum power experienced by the fuel, $q_{M}^{\prime}$ in kW/m, and $n_{f}$ is the total number of fragments expected taking into account the average burnup of the fuel rod given by $B u_{a v}$ in $\mathrm{MWd} / \mathrm{kgHM}$. Once the number of fragments are known the characteristic length of the fragments is calculated via:

$l_{f}=D_{F P} \min \left(1, \frac{\pi}{n_{f}}\right)$

where $l_{f}$ is the characteristic length in $\mathrm{m}$ and $D_{F P}$ is the as-fabricated fuel pellet diameter in $\mathrm{m}$. Limited experimental data exists for the size of fuel particles formed during pulverization. Pulverization of the fuel is hypothesized to be caused by overpressurization of gas bubbles in the highly porous high burnup structure that forms at the pellet periphery as irradiation progresses. The overpressurization and rupture of these bubbles results in the porous region of fuel disintegrating into very fine particles. Turnbull et al. [8] have developed a pulverization threshold that is a local temperature function of local burnup as shown in Figure 1. Jernkvist and Massih argue that even if the threshold has been exceeded and there is a contact pressure between the fuel and cladding of greater than $50 \mathrm{MPa}$ pulverization will not occur.

Once the volume of the pellet subjected to pulverization in a layer is determined the mass fraction of pulverized fuel $\left(x_{p}\right)$ is calculated knowing the total volume of fuel in a particular layer. The mass fraction of fragments $\left(x_{f}\right)$ is simply given by $x_{f}=1-x_{p}$. Given the known mass fractions the packing fraction can be calculated by solving the following set of equations using an internal newton solve to iterate to convergence within a specified tolerance.

$a^{2}+2 G a b+b^{2}=1$

$a=\frac{\phi_{p}\left(\phi_{f}-x_{f} \phi\right)}{\phi \phi_{f}}$ 


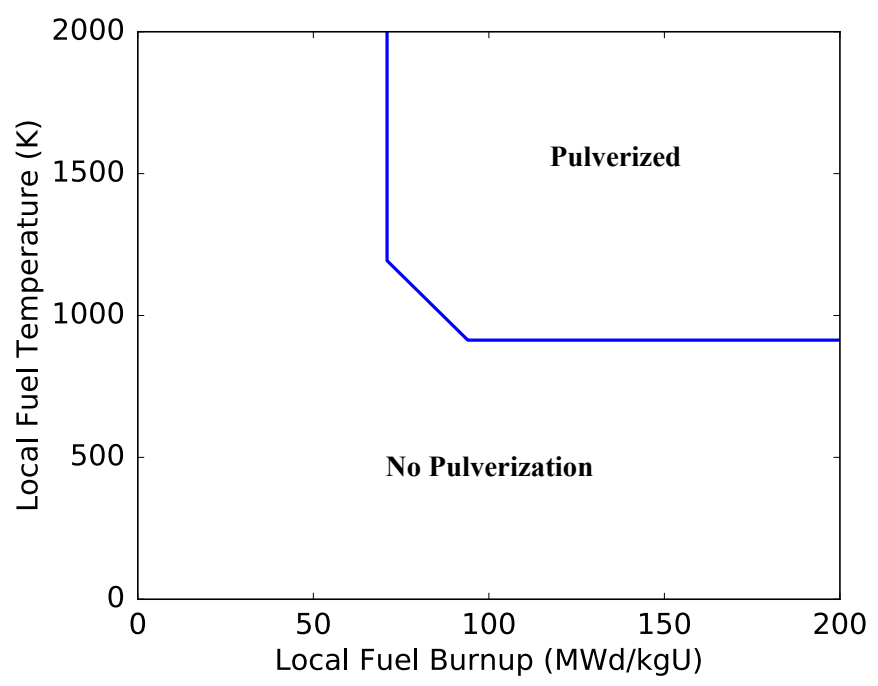

Figure 1: Burnup dependent temperature threshold for fuel pulverization [6] 8].

$b=\frac{\phi_{p} \phi_{f}-\phi \phi_{f}\left(x_{p}+x_{f} \phi_{p}\right)}{\phi \phi_{p}\left(1-\phi_{f}\right)}$

$G= \begin{cases}0.738\left(D_{p}^{p} / D_{p}^{f}\right)^{-1.566}, & D_{p}^{p} / D_{p}^{f} \leq 0.824 \\ 1, & D_{p}^{p} / D_{p}^{f}>0.824\end{cases}$

$D_{p}=\left(3.9431-\frac{4.5684}{\psi}+\frac{1.8660}{\psi^{2}}\right) V_{p}^{1 / 3}$

where $a, b$, and $G$ are unitless parameters, $\phi_{p}$ (default value of 0.72 ) and $\phi_{f}$ (default value of 0.69) are the packing fractions assuming the crumbled bed of fuel particles is entirely made up of pulvers or fragments respectively, $\phi$ is the packing fraction of the binary system (what is being solved for), $D_{p}^{p}$ and $D_{p}^{f}$ are the equivalent packing diameters of the pulvers and fragments, and $\psi$ and $V_{p}$ are the sphericity and volume of a particular particle shape. Jernkvist and Massih suggest that fragments are treated as prismatic and pulvers as octahedral. Thus for fragments, $\psi=0.716$ and $V_{p}=0.4330 l_{f}^{2}$ where $l_{f}$ is the characteristic length calculated from Equation 3 For pulvers, $\psi=0.846$ and $V_{p}=0.4714 l_{p}^{3}$ where $l_{p}$ is the characteristic length of the pulver (default value of $100 \mu \mathrm{m}$ ). The above algorithm is solved for each layer.

Once the packing fraction is obtained the rest of the axial relocation algorithm can be completed including mass relocation, thermal conductivity degradation, increase of effective diameter of the porous bed of fragments in layers that have crumbled for heat redistribution, and internal volume calculations upon fuel crumbling. The condition on fuel crumbling in a given layer is given by

$m_{k}^{M}>m_{k}^{i}$

where $m_{k}^{i}$ represents the initial mass in the k:th layer and $m_{k}^{M}$ represents the mass in the layer if it is completely filled with crumbled fuel given by

$m_{k}^{M}=\phi_{k} \rho_{f} \pi L_{k} R_{c i k}^{2}$ 
where $\phi_{k}$ is the packing fraction for the k:th layer, $\rho_{f}$ is the fuel density, $L_{k}$ is the length of the k:th layer and $R_{c} i k$ is the inner cladding radius in the $\mathrm{k}$ :th layer. In addition to the condition of crumbling four additional constraints are required for realistic and numerical reasons. The first two constraints prevent fuel from relocating upwards within the fuel rod and limit the amount of fuel that can relocate to the total amount of existing mass in layers above a given layer. These lower $\left(m_{k}^{L}\right)$ and upper $\left(m_{k}^{U}\right)$ constraints are given by

$m_{k}^{L}=\sum_{j=1}^{k} m_{j}^{o}-\sum_{j=1}^{k-1} m_{j}$

$m_{k}^{U}=m_{k}^{r}+\sum_{j=1}^{k} m_{j}^{o}-\sum_{j=1}^{k-1} m_{j}$

where the $m_{j}^{o}$ represents the mass in the $\mathrm{j}$ :th layer at the beginning of the timestep $\left(t_{o}\right), m_{j}$ represents the current mass in the j:th layer and $m_{k}^{r}$ represents the available mass to be relocated into the k:th layer. The two other constraints assume a small fraction of the initial fuel mass in a layer will remain in that layer (i.e., stuck to the cladding) which is denoted by $x^{r}$ with a default value of 0.01 and the cladding distention must be sufficiently large in a layer to generate a fuel-to-cladding gap that will accommodate fuel movement (denoted by $g^{\text {th }}$ with a default value of $0.2 \mathrm{~mm}$ ). The mass relocation algorithm given the above definitions and constraints is divided into two loops. The first loop iterates from the top of the fuel downward determining the amount of fuel that can be relocated into a particular layer. The second updates the mass in the layers while enforcing the lower and upper mass constraints by iterating from the bottom of the fuel stack upward. These loops are illustrated in Figures 2 and 3 .

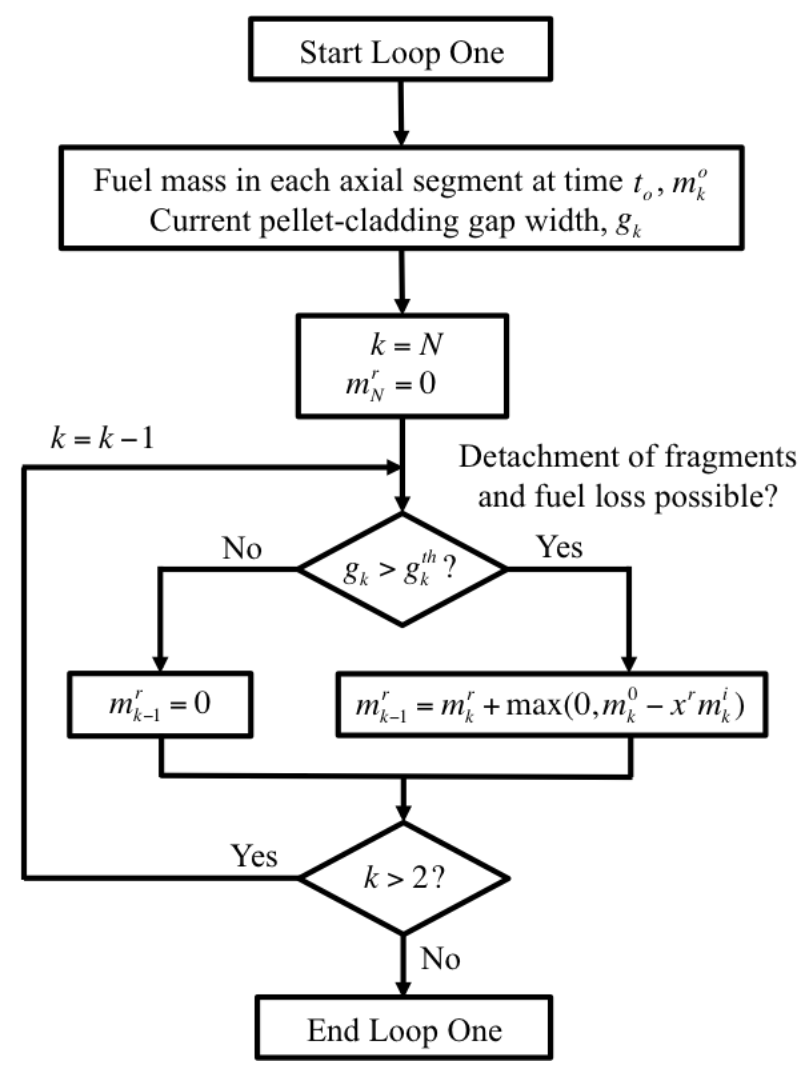

Figure 2: The first loop of the axial relocation algorithm which determines the amount of relocatable mass $\mathrm{m}^{r}$ that can be accommodated in each layer. Adapted from [6]. 


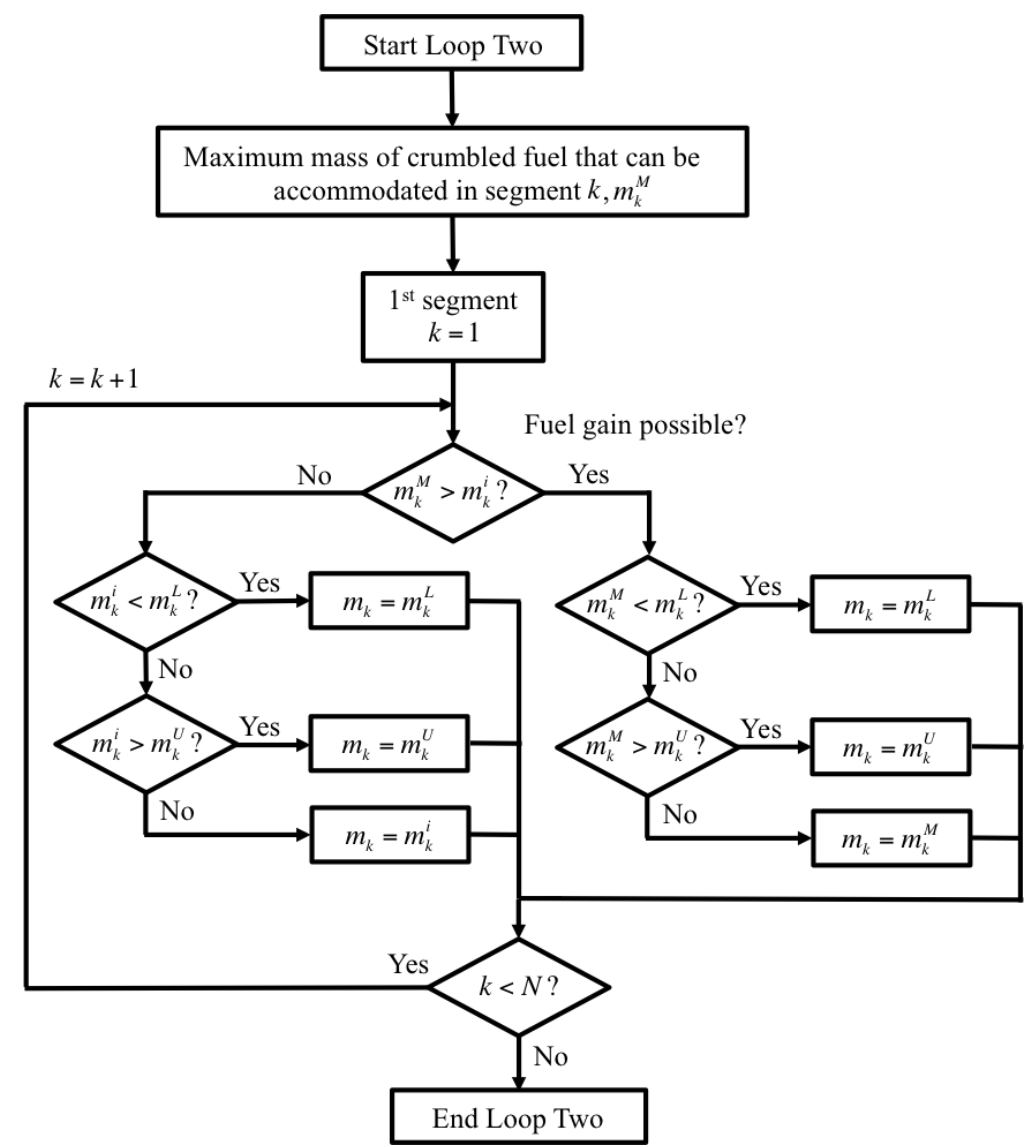

Figure 3: The second loop of the axial relocation algorithm that enforces the constraints and moves the mass to the appropriate layers. Adapted from [6].

The relocation of fuel throughout the rod during the LOCA transient results in a redistribution of the energy generation. In a layered $1 \mathrm{D}$ representation only the radial direction is of great importance in the heat conduction equation. The modified heat conduction in the radial equation is given by:

$\phi \rho_{f} c_{p f} \frac{\partial T}{\partial t}-\frac{1}{r^{\prime}} \frac{\partial}{\partial r^{\prime}}\left(k_{e f f} r^{\prime} \frac{\partial T}{\partial r^{\prime}}\right)=\phi q^{\prime \prime \prime}$

where $c_{p f}$ is the specific heat of the fuel. Care must be taken to ensure that in layers where the fuel is crumbled that the outer radius of the fuel is moved outward towards the cladding to take into account the increase in effective diameter of the porous bed of fuel fragments. In the model, Jernkvist and Massih assume that a residual fuel-to-cladding gap ( $g^{r}$ which is a model parameter with a default value of $2 \mu \mathrm{m}$ ) remains in the crumbled layers (illustrated in Figure 4 adapted from [6]). In layers partially or completely void of fuel the original radial position is used along with the $k_{f}$ instead of $k_{e f f}$. The correlation used to calculate $k_{e f f}$ is that by Chiew and Glandt [9]. 

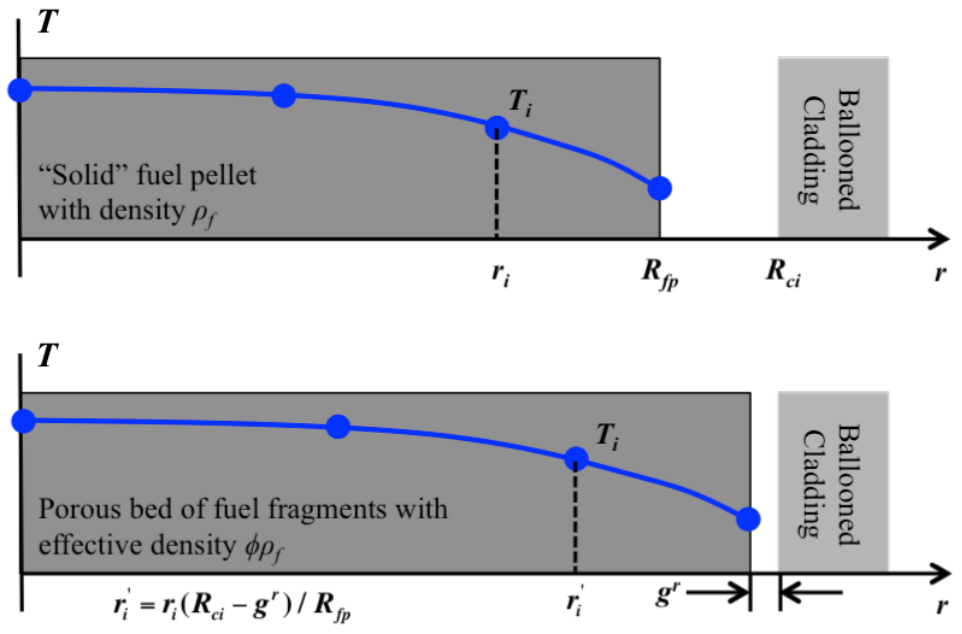

Figure 4: Change in fuel geometry and effective fuel density following fuel pellet collapse in the ballooned region of the cladding. A residual gap $g^{r}$ is assumed to remain. 


\section{The Extension of the Axial Relocation Model}

The Jernkvist and Massih axial relocation model provides a solid foundation for axial relocation fuel modeling. The original development based upon a layered 1D framework allows for easy tracking of the mass in each of the discrete layers. Unfortunately, during LOCA conditions the thermal degradation on the waterside surface of the cladding is usually non-uniform in the azimuthal direction which the layered 1D formulation formulation cannot capture due to its axisymmetric assumption. Thus, when extending the axial relocation model to two dimensions including azimuthal effects is of great importance. This importance eliminates the possibility of extending the model to 2D axisymmetric simulations due to the inherent symmetry and the lack of discrete layers to account for the mass movement. Moreover, as the number of layers increase in a layered 1D analysis the results approach that of a 2D axisymmetric simulation. To overcome these deficiencies of extending the model to the 2D-RZ formulation a new 2D framework called layered 2D has been developed. A brief overview of this approach is described in Subsection 3.1 The other subsections in this section describe the necessary developments to extend the original Jernkvist and Massih axial relocation model to work in the new layered 2D framework including the relocatable mass threshold, the calculation of cladding volume, and the movement of the mesh for heat conduction purposes. Even with these extensions the axial relocation model continues to provide the exact same solutions as before when used with a layered 1D representation of the fuel rod.

\subsection{Layered Two-Dimensional Framework}

The layered 2D framework that was used to facilitate the axial relocation model extension was developed by the author of this report as part of his $\mathrm{PhD}$ research at the University of South Carolina. This framework, like the layered 1D framework, models the fuel rod with a number of discrete axial slices. The difference being each layer is represented by a $2 \mathrm{D}$ slice containing a fuel disc, gas gap ring, and a cladding ring. Figure 5 illustrates the $2 \mathrm{D}$ formulation. The left figure shows the axial discretization of the rod which is similar to the layered 1D discretization [10] except azimuthally symmetry is not assumed (i.e., symmetry is not assumed at the rod centerline). The right figure provides a top view of a single axial slice (highlighted in red in the left figure). The thermo-mechanics of each axial layer is solved as a separate generalized plane strain calculation with global parameters such as fission gas release, rod internal pressure, and axial (out of plane) strain being coupled between the layers to allow predictions of these global rod parameters as well as others including fuel and cladding elongation. The layered 2D approach can be used with the out of plane direction in any of the coordinate directions.
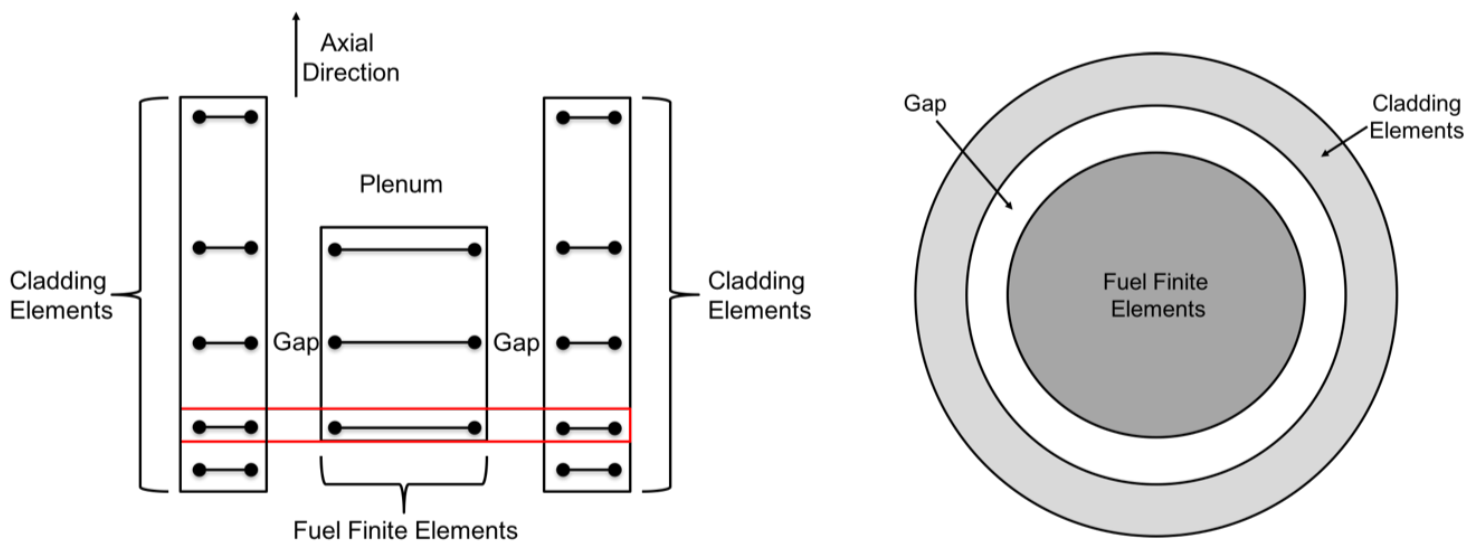

Figure 5: Schematic describing the layered 2D formulation. The axial discretization of the fuel rod including fuel gap and cladding elements where azimuthal symmetry is not guaranteed (left). The top view an axial slice with fuel, gap and cladding shown (right).

\subsection{Gap Calculation in Relocatable Mass Loop}

In the layered 1D formulation the gap in each layer that is compared to the minimum gap threshold (denoted as $\left.g_{k}^{t h}\right)$ in Figure 2 is simply calculated as the difference between inner cladding radius at time $t$ and the as-fabricated fuel radius. 
If the calculated gap exceeds the minimum gap threshold mass is deemed relocatable from the layer. In the layered 2D formulation the possibility of azimuthally varying gap thicknesses required the development of a new UserObject in Bison that calculates the maximum inner cladding radius in each layer. Subtracting the as-fabricated fuel radius from this maximum inner cladding radius determines the gap size compared to $g_{k}^{\text {th }}$. Therefore, in the extended axial relocation model if any azimuthal location has a fuel-to-clad gap greater than the threshold gap (default is $0.2 \mathrm{~mm}$ ) mass is deemed relocatable in that layer.

\subsection{Cladding Volume}

Similarly to the gap calculation described above the layered internal cladding volume in each layer is easily calculated in the layered 1D formulation due to the azimuthal symmetry. In that case the current cladding volume used in the calculation of crumbled mass $m_{k}^{M}$ (See Eq. 10 is simply given by $\pi L_{k} R_{c i k}^{2}$. In the layered $2 \mathrm{D}$ formulation azimuthal variation in cladding distention can lead to a non-circular cross section of the inner area of the cladding. Therefore, a new Bison UserObject was developed to compute the cladding internal volume on a layered basis. The volume of the enclosed space in each layer is calculated as the integral over the cladding inner surface in that layer. The integral calculation allows for arbitrary cladding balloons to be represented. It should be noted that due to finite element limitations the accuracy of the integral depends upon the density and order of the finite element mesh as curved domains may not be exactly captured due to faceting.

\subsection{Mesh Movement in Crumbled Layers}

The last portion of the existing axial relocation model that needed to be extended to work in the layered 2D framework is the movement of the fuel mesh towards the cladding in crumbled layers of fuel for thermal feedback and heat generation purposes. In the layered $1 \mathrm{D}$ formulation the approach was simply to calculate an eigenstrain that would move the current position of the outer fuel radius to a position that is within the residual gap $\left(\mathrm{g}^{r}\right)$ of the cladding as seen in Figure 4 The eigenstrain is calculated as a true strain via:

$\epsilon=\ln \left(1.0+\frac{R_{c i}-g^{r}-R_{p o}}{R_{p o}}\right)$

where $R_{c i}$ is the current inner cladding radius, $g^{r}$ is the residual gap, and $R_{p o}$ is the outer fuel radius from the timestep in which the layer was first calculated to be crumbled. In the layered 2D framework the eigenstrain calculation above is still used. However, because of the potential variability in the cladding radius azimuthally the eigenstrain could also potentially vary azimuthally. Thus, a new UserObject was developed that stores the calculated eigenstrain as a function of axial layer and azimuthal position. Then, a material property queries the UserObject to determine the eigenstrain to be applied at any position throughout the mesh. The new approach allows asymmetric balloons to fill in to within the residual gap at all locations in layers that have crumbled. 


\section{Bison Demonstration Cases}

To demonstrate the benefit of extending the axial relocation model to accommodate the important azimuthally varying boundary conditions present during LOCA transients two sets of simulations have been completed: azimuthally symmetric and asymmetric. The azimuthally symmetric cases are to demonstrate that the existing and extended axial relocation models predict the same behavior under symmetric loading conditions. The asymmetric case is used to demonstrate the significant difference between the extended model and existing model predictions due to the existing model being unable to capture asymmetric features due to the inherent symmetry in the layered 1D formulation on which the existing axial relocation model is based.

In the original report by Jernkvist and Massih [6] two verification cases were included for the mass relocation loops shown in Figures 2 and 3 . Here, similar cases were used to confirm that the layered 1D and layered 2D approaches provide the same results under azimuthally symmetric balloons. This ensures that the extensions described in Section 3 are correctly implemented before examining asymmetric balloons. The two cases analyzed here are known as single balloon and twin balloon. In both test cases the active length of the fuel is $\left(L_{a}=1.8 \mathrm{~m}\right)$ with a fuel pellet diameter of $9.0 \mathrm{~mm}$. The initial fuel-to-cladding gap is assumed to be zero (i.e., the gap is closed). The effective packing fraction is assumed to be 0.75 after fuel crumbling and 18 equal length axial segments are used. The duration of the simulation is $100 \mathrm{~s}$. The single balloon verification test is to simulate cladding distention that is maximum at the midplane of the active length $(z=0.9 \mathrm{~m})$. The twin balloon verification test is to simulate the effect of having a spacer-grid at the midplane of the active length. In the single balloon case the inner cladding radius is varied by:

$$
R_{c i}(t, z)=4.5 \times 10^{-3}+1.0 \times 10^{-5} t \sin \left(\frac{\pi z}{L_{a}}\right)
$$

and in the twin balloon case the inner cladding radius is varied by:

$R_{c i}(t, z)=4.5 \times 10^{-3}+1.0 \times 10^{-5} t\left|\sin \left(\frac{2 \pi z}{L_{a}}\right)\right|$

The results of these verification cases are illustrated in Figures 6 and 7 . Each figure contains three subfigures that provide snapshots in time $(60,80$, and $100 \mathrm{~s})$ of the fuel mass fraction within the rod. A fuel mass fraction greater than one means the region has gained mass. Mass loss occurs in regions with a value less than one. In the single balloon case as time progresses the amount of fuel loss at the top of the rod increases and moves into the midplane of the rod as expected. As observed in some experiments, and captured by the model, a fuel plug at the top of the rod where fuel loss may not occur is predicted. In the twin balloon case mass loss is observed from two locations including the top portion of the rod and just below the spacer grid at the midplane of the rod. This mass relocates into the two balloons.

Since the extended axial relocation model applied to both layered 1D and layered 2D geometries with symmetric cladding balloons have been verified to yield identical results, confidence can be had that the layered $2 \mathrm{D}$ formulation and all of the required extensions have been implemented correctly. Now, consider the previous single balloon case but with an azimuthal variation added to the inner cladding displacement as given below:

$R_{c i}(t, \theta, z)=4.5 \times 10^{-3}+1.0 \times 10^{-5} t \sin \left(\frac{\theta}{2}\right) \sin \left(\frac{\pi z}{L_{a}}\right)$

The above equation has the same maximum displacement as the symmetric balloon case but it only occurs at an angle of $\pi$. The mass fraction results of this case compared to the symmetric balloon for layered 1D as given by Equation 15 (since the layered 1D simulation does not support azimuthal variation) are shown in Figure 8 It is observed that even though the maximum balloon size (i.e., the same maximum cladding displacement) is the same, the asymmetric ballooned rod observes zero mass relocation throughout the simulation. Less mass movement occurs because the overall internal volume in the ballooned layers available to accommodate additional mass is smaller than the symmetric case (See Figure 9). 


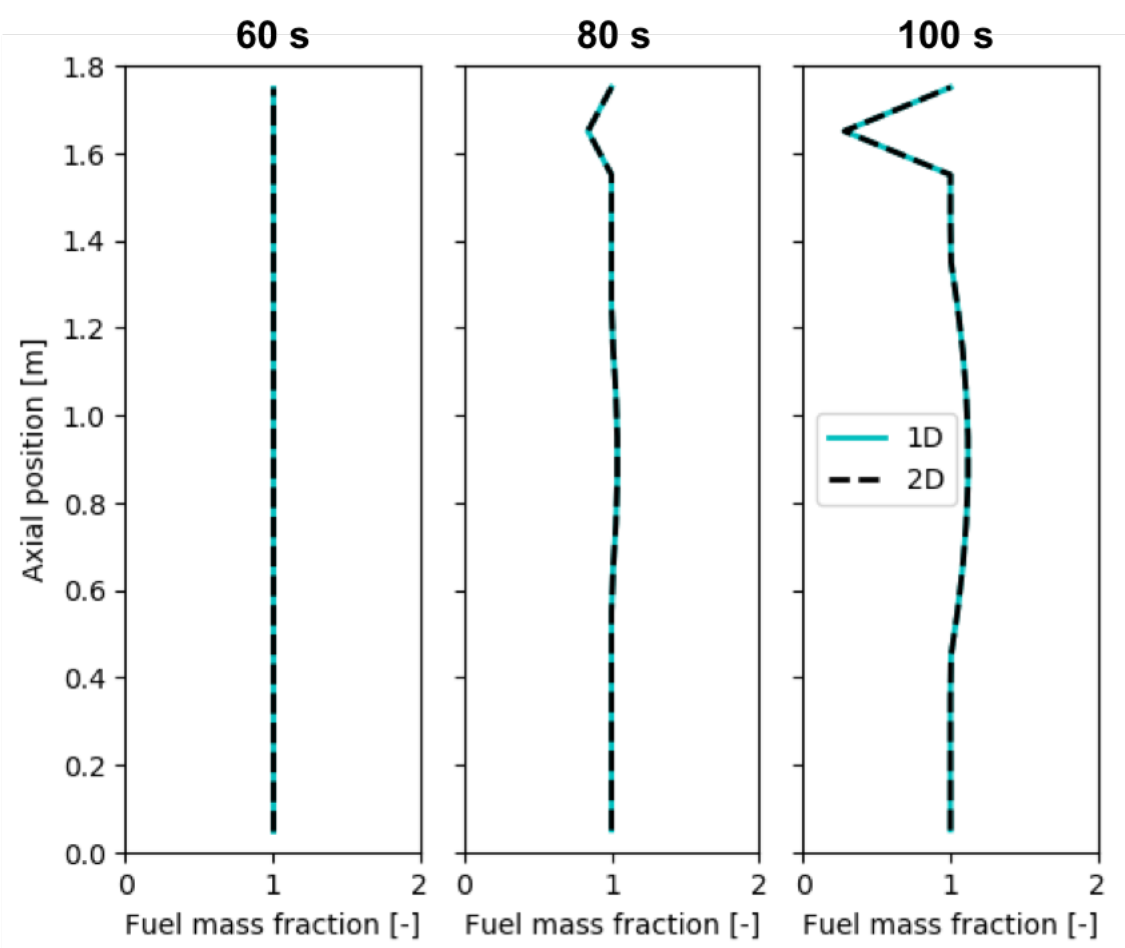

Figure 6: Fuel mass fraction as a function of axial position for a symmetric single balloon case at selected snapshots in time of 60 , 80, and $100 \mathrm{~s}$. Layered $1 \mathrm{D}$ and layered 2D formulations are compared.
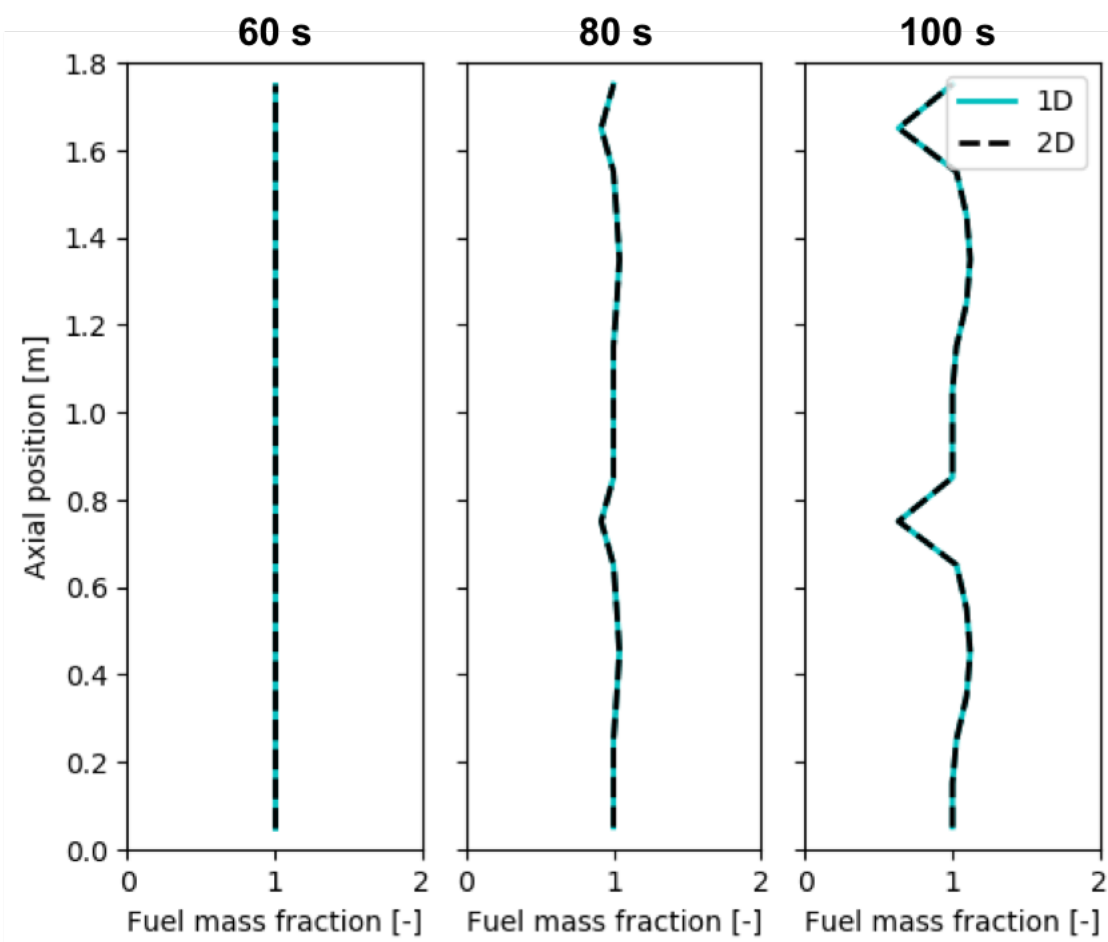

Figure 7: Fuel mass fraction as a function of axial position for a symmetric twin balloon case at selected snapshots in time of 60, 80, and $100 \mathrm{~s}$. Layered 1D and layered 2D formulations are compared. 

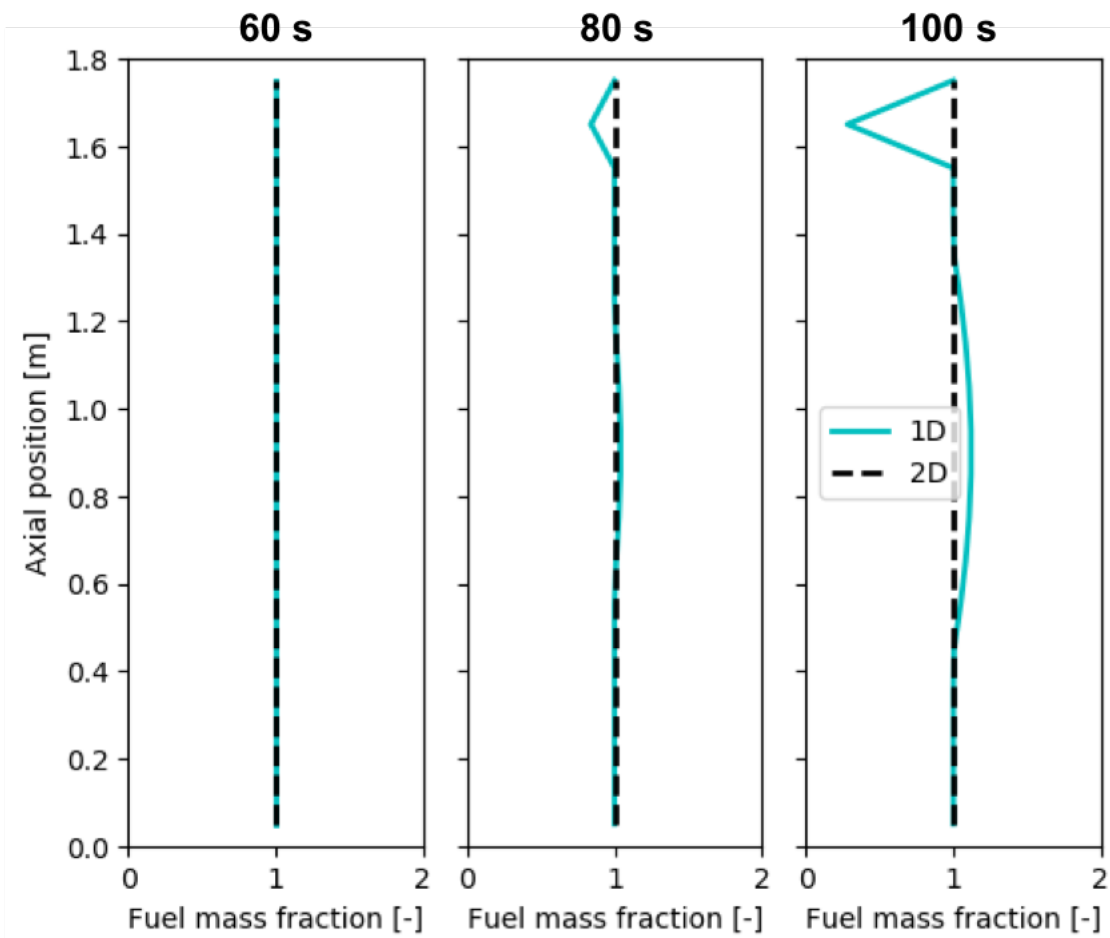

Figure 8: Fuel mass fraction as a function of axial position comparing the symmetric (1D) and asymmetric (2D) single balloon cases at selected snapshots in time of 60,80, and $100 \mathrm{~s}$ to illustrate the importance of capturing azimuthal effects.
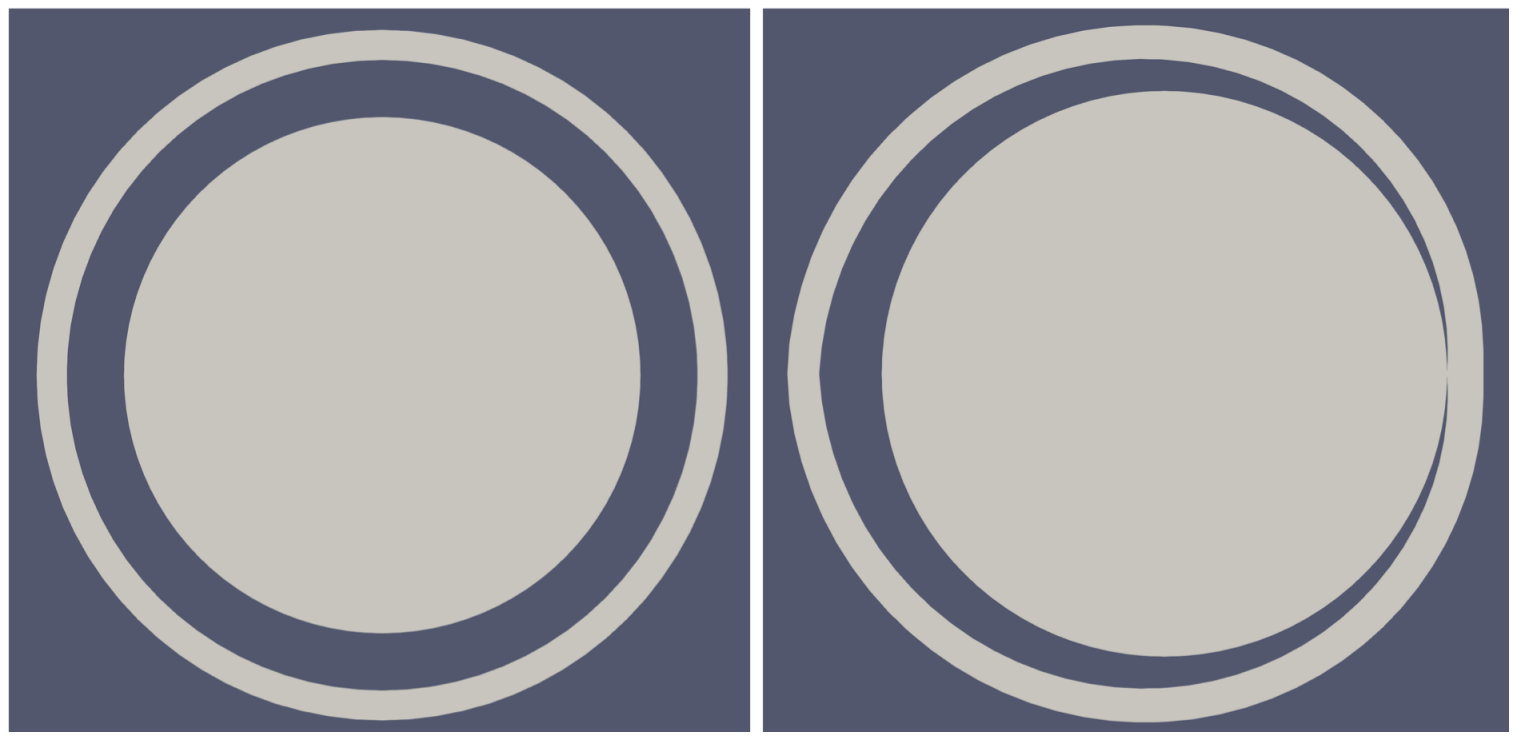

Figure 9: A top view of the ninth axial layer from the bottom of the rod at 100 seconds for the symmetric (left) and asymmetric (right) single balloon cases. 


\section{Summary}

The existing axial relocation model implemented in Bison in FY17 using the layered 1D formulation has been extended to two dimensions using a new layered 2D formulation. This approach allows for the inclusion of the important azimuthal variations in boundary conditions present during LOCA conditions allowing for the prediction of asymmetric cladding balloons that are observed experimentally. The axial relocation model required new capabilities to be added to Bison to be able to work with this new framework including the gap calculation in the relocatable mass loop, the calculation of the cladding internal volume for each layer, and the algorithm for determining the eigenstrain required to move the mesh after crumbling for thermal feedback purposes. Demonstration cases were provided that verify that the layered 1D and layered 2D formulations predict the same axial distribution of mass when the cladding balloon is azimuthally symmetric. An additional case was included that highlights the significantly different fuel mass fraction predictions when azimuthally asymmetric cladding distention occurs including the possibility of no fuel relocation. Additional work is required to validate the extended axial relocation model to experiments containing azimuthally varying boundary conditions. The behavior of the axial relocation model could be extended further by implementing the fragmentation model by Brankov [11] that allows for more than a binary system of particle sizes for determining the fuel packing fraction. 


\section{Acknowledgements}

This work was funded by the U.S. Department of Energy under the Consortium for Advanced Simulation of Light Water Reactors (CASL). The conference paper has been authored by a contractor of the U.S. Government under Contract DE-AC07-05ID14517. Accordingly, the U.S. Government retains a non-exclusive, royalty free license to publish or reproduce the published form of this contribution, or allow others to do so, for U.S. Government purposes. 


\section{References}

[1] H. Sonnenburg, W. Wiesenack, J. Karlsson, J. Noirot, V. Garat, N. Waeckel, F. Kattout, A. Cabrera-Salcedo, J. Zhang, G. Khvostov, A. Gorzel, V. Brankov, F. Nagase, P. Raynaud, M. Bales, T. Taurines, T. Nakajima, and A. Alvestav. Report on fuel fragmentation, relocation, and dispersal. Technical Report NEA/CSNI/R(2016)16, Organisation for Economic Co-operation and Development Nuclear Energy Agency Committee on the Safety of Nuclear Installations, 2016.

[2] J. D. Hales, S. R. Novascone, B. W. Spencer, R. L. Williamson, G. Pastore, and D. M. Perez. Verification of the BISON fuel performance code. Annals of Nuclear Energy, 71:81-90, 2014.

[3] R. L. Williamson, J. D. Hales, S. R. Novascone, M. R. Tonks, D. R. Gaston, C. J. Permann, D. Andrs, and R. C. Martineau. Multidimensional multiphysics simulation of nuclear fuel behavior. Journal of Nuclear Materials, 423:149-163, 2012.

[4] R. L. Williamson, K. A. Gamble, D. M. Perez, S. R. Novascone, G. Pastore, R. J. Gardner, J. D. Hales, W. Liu, and A. Mai. Validating the BISON fuel performance code to integral LWR experiments. Nuclear Engineering and Design, 301:232 - 244, 2016.

[5] L. J. Siefken. Fuel axial relocation in ballooning fuel rods. In Proceedings of the International Conference on Structural Mechanics in Reactor Technology, Chicago, IL, USA, 1983.

[6] L. O. Jernkvist and A. Massih. Model for axial relocation of fragmented and pulverized fuel pellets in distending fuel rods and its effects on fuel rod heat load. Technical Report SSM-2015:37, Strål säkerhets myndigheten, 2015.

[7] O. Coindreau, F. Fichot, and J. Fleurot. Nuclear fuel rod fragmentation under accidental conditions. Nuclear Engineering and Design, 255:68-76, 2013.

[8] J. A. Turnbull, S. K. Yagnik, M. Hirai, D. M. Staicu, and C. T. Walker. An assessment of the fuel pulverization threshold during loca-type temperature transients. Nuclear Science and Engineering, 179:477-485, 2015.

[9] Y. C. Chiew and E. D. Glandt. The effect of structure on the conductivity of a dispersion. Journal of Colloid and Interface Science, 91(1):90-104, 1983.

[10] S. A. Pitts, S. R. Novascone, H. Chen, B. W. Spencer, S. Satpathy, R. J. Gardner, and J. D. Hales. Verify and validate 1.5D capability. Technical Report CASL-U-2017-1380-000, Idaho National Laboratory, June 2017.

[11] V. V. Brankov. Modelling of fuel fragmentation, relocation and dispersal during Loss-of-Coolant Accident in Light Water Reactor. PhD thesis, École Polytechnique Fédérale de Lausanne, October 2017. 